\title{
Preface - Advances in CKD 2018
}

Renal Research Institute's 20th International Conference on Dialysis, Advances in Chronic Kidney Disease (CKD) in Orlando, FL, is reaching a milestone by celebrating its 20th birthday. As in previous years, the conference highlights the latest scientific developments in kidney research, spanning from basic science to clinical nephrology, acute and CKDs, hemodialysis and peritoneal dialysis (PD) management, kidney transplantation, and epidemiology.

This year we have expanded the scope of the topics and our audience, by including a hands-on peripheral vascular disease ultrasound workshop and a Nurse Care Team Track, addressing topics of interest to renal nurses and shared topics with nephrologists.

The special issue of Blood Purification includes an update on the pathophysiology of arterial stiffness by London et al. Premature vascular aging is the most characteristic arterial change observed in end-stage renal disease (ESRD). Arterial stiffening is more pronounced in the aorta than in peripheral arteries and observations suggest a "pressure independent stiffening" that present treatments can only modestly prevent.

The deleterious effects of fluid overload on cardiovascular outcomes are well known. In a review by Dekker et al., we learn that there is an additive increased mortality risk when both fluid overload and inflammation are present. The increased mortality appears to persist - albeit to a lesser degree - even shortly after resolution of both risk factors.
Nerbass et al. discuss what they call "salty relationship" of the mechanisms involved in sodium intake and blood pressure in patients with CKD. Indeed, dietary sodium intake, which has been associated with numerous modifiable risk factors for cardiovascular disease in CKD patients and salt sensitivity, is one of the main determinants of blood pressure response. Furthermore, markers of kidney damage, such as proteinuria, are also associated with high sodium intake and are the key risk factors for subsequent all-cause and cardiovascular mortality.

Van de Wal-Visscher et al. offer a thorough review of the importance of magnesium and its many roles in various processes such as neuromuscular and neurotransmitter release, mineral bone metabolism, energy metabolism, regulation of vascular tone, heart rhythm, and platelet-activated thrombosis. The authors also discuss new research into dialysate magnesium levels.

Lin et al. present case studies that provide interesting and practical insights into the management of acid-base and electrolytes in CKD and ESRD patients. Each case report comes with recent investigational results integrated into the existing body of knowledge, and illustrates pathophysiology, diagnosis, and treatment options. For example, a case of metabolic acidosis, where compensatory mechanisms to combat daily acid load can cause sustained inflammation and renin-angiotensin-aldosterone system activation, accelerate $\mathrm{CKD}$ progression and increasing mortality. 
The paper by van de Velde-Kossmann highlights the importance of examining skin manifestations in ESRD patients, which could range from nonspecific pigmentary changes, such as hyperpigmentation, pallor, a slate-grey discoloration, and a yellow skin hue, to specific changes associated with diabetes, connective tissue disorders, vascular disorders, and genetic disorders, for example, angiokeratomas in Fabry's disease.

While pregnancy is uncommon in women with ESRD, when it occurs, fetal and maternal complications remain high. Maternal and fetal outcomes are best with an experienced multidisciplinary care teams, as expressed by Tangren et al. in their paper on pregnancy and ESRD, where they discuss the various stages and management interventions.

Yousif et al. present the results of their original investigation on acute kidney injury (AKI) from a single center experience from Khartoum, Sudan. AKI is commonly caused by community-acquired single diseases such as malaria, post-diarrheal hemolytic uremic syndrome, diarrhea, sepsis, and various forms of poisoning. The authors found that only $38 \%$ of patients recovered renal function, rendering it a major risk factor for later progression to ESRD. In the light of the high costs of renal replacement therapies, AKI prevention is crucial to decrease its severe impact on morbidity and mortality, and health costs. Inexpensive, simple interventions such as oral rehydration and fluid therapy may help to prevent AKI.

The papers by Lau et al. and Hruskova et al., respectively, provide the latest diagnostic and therapeutic options for the elderly population, whose decline in renal function has been associated with aging and chronic conditions such as diabetes, hypertension, and atherosclerotic and vascular disease. However, a significant proportion is due to a potentially treatable disease such as the antineutrophil cytoplasmic antibodies-positive rapidly progressive-positive glomerulonephritis, or anti-neutrophil cytoplasmic antibodies-associated vasculitis, which is the most common etiology in the very elderly. Both papers are timely because "as the population world-wide ages, the epidemic of kidney disease will also increase" as expressed by Lau et al.

Starting from current PD, Girsberger et al. look ahead on novel options of PD utilization. The rationale of using continuous ultrafiltration through PD in the treatment of acute or chronic heart failure complicated by kidney malfunction as in the cardio-renal syndrome. Additionally, usage of PD benefits cardio-renal syndrome patients with refractory volume overload without ESRD. The authors describe also recent developments in PD fluids, such as the hyperbranched polyglycerol, which showed better preservation of ultrafiltration and fewer toxic effects in rodent models.

In their systematic review about home hemodialysis (HHD), Miller et al. conclude that two-thirds of the included publications demonstrate improved clinical outcomes in patients who chose HHD over in-center dialysis, including improved survival, cardiovascular, nutritional, and quality of life parameters. The authors recommend that HHD should be considered as a modality choice for substantially more than the current $1.8 \%$ of dialysis patients in the US.

Van der Sande et al. provide an up-to-date review of the pathogenesis and prevention of intra-dialytic hypotension (IDH), a topic as current as ever. The authors suggest that IDH-associated changes in perfusion of vital organs such as heart, gut, and brain are related to short- or long-term complications. Therefore, the authors recommend routine on-line measurement of tissue perfusion for the prevention of IDH, along with currently used preventive measures.

The interesting investigation of Han et al. is one of the latest contributions to studies on HD patients' physical activity monitored by wearable trackers. The findings identify for the first time that the walkability of a neighborhood is positively correlated to and associated with measured physical activity levels in HD patients. The study opens possibilities for further novel investigations into physical activity of ESRD patients and the environment that surrounds them.

Patel et al. report results from the Monitoring Dialysis Outcomes database. Their retrospective cohort analysis included 23,353 HD patients from 4 continents and explored the association between heights and outcomes. Unlike patients in North-America, taller patients have increased risk of mortality in Europe, Asia Pacific, and Latin America.

The review by Cooper et al. provides an update on kidney xenotransplantation. The authors provide a detailed analysis of the major immune pathobiological barriers and present the status of pig genetic manipulation, with some encouraging results, which may foster the initiation of clinical trials using non-human primates.

The paper from Nalesso et al. on standardization of protocols for hemodialysis vascular access assessment underlines the importance of using ultrasound and color Doppler. The key message is that the nephrologist must be acquainted with this important tool and cannot delegate any longer this procedure of ultrasound assessment of vascular access for dialysis to other specialists. Along 
the same lines, the nephrologist should be in charge of new developments in the area of vascular access and one of them is the new plastic cannulas used by the authors in the last paper of this collection. A complete description of the available technologies is also provided.

Finally, the research group of Vicenza present the result of a routine adoption of biomarkers of AKI in clinical practice. The possibility to identify patients at risk and make an accurate assessment of the patient profile thanks to the use of biomarkers is underlined by the interesting predictive ability of these new diagnostic tools also in view of follow-up and kidney function after the episode of acute illness.

Looking back on a history of 20 years, the Renal Research Institute continues to be dedicated to advancing therapy options for dialysis patients and to provide the highest caliber of care based on research and advanced clinical technology. Renal Research Institute is committed to research and innovation that will lead to improved patient outcomes and quality of life. It has active national and international research programs with strong academic alliances with the University of Michigan, Ann Arbor, MI, USA; University of North Carolina, Chapel Hill, NC, USA; University of Rochester, Rochester, NY, USA; Icahn School of Medicine at Mount Sinai, New York, NY, USA; St. Raphael Hospital/Yale, New Haven, CT, USA; University of Massachusetts, Lowell, MA, USA; Pontifical Catholic University of Paraná, Curitiba, Brazil; the University of Guadalajara, Mexico; the University of Maastricht, The Netherlands; the Taipei Medical University Hospital, Taiwan; the University of Konstanz, Germany; and the University of Graz, Austria, among others.

We hope that you will enjoy the wide range of papers published in this issue of Blood Purification and that you will consider attending next year's 21st International Conference on Dialysis, Advances in Kidney Disease 2019, which will take place in New Orleans.

Peter Kotanko, New York, NY, USA Laura Rosales, New York, NY, USA Claudio Ronco, Vicenza, Italy 